\title{
237
}

\section{PARTICLE ACCELERATION BY THE SUN}

\author{
Rapporteur paper for the 19th International Cosmic Ray Conference
}

\author{
R. P. Lin \\ Space Sciences Laboratory, University of California, Berkeley, CA 94720
}

\section{Introduction}

Large solar flares are the most energetic natural particle accelerators in the solar system, occasionally accelerating ions to many $\mathrm{GeV}$ and electrons to $z 10^{2} \mathrm{MeV}$ energies. Radio, X-ray and gamma-ray, and energetic particle observations obtained before the 1980 solar maximum suggest that there are at least two acceleration processes associated with solar flares. During the impulsive or flash phase electrons are of ten accelerated to $\sim 10-100 \mathrm{keV}$ energies, even in small flares or subflares. For some flares, the energy contained in these electrons may be a substantial fraction of the total flare energy. Thus the primary energy release mechanism for flares may initially convert stored magnetic energy in to energetic electrons. These electrons can produce most of the observed impulsive phase flare phenomena through their interactions with the solar atmosphere. In large flares a second acceleration sometimes occurs which accelerates both ions and electrons to $\mathrm{MeV}$ energies and above. This second acceleration appears to have a close association with shock waves in the solar atmosphere as observed by type II radio bursts. Based on observations of escaping particles and hard $\mathrm{X}$-ray and gamma ray bursts, the impulsive phase acceleration events occur on the order of $\sim 10^{2}$ time a month near solar maximum while large solar energetic particle (LSEP) events where $>10 \mathrm{MeV}$ ions and relativistic electrons are accelerated to observable levels occur a few times a mon th.

Gamma-ray observations from the SMM spacecraft in this solar maximum, however, indicate that the delays between electron and ion acceleration can sometimes be very short, $\sim 1 \mathrm{~s}$, so the separation in to two types of acceleration may not be meaningful. Furthermore, the relationship between the solar energetic particles observed in interplanetary space and those which produce gamma-ray and neutrons at the Sun is unclear. Often intense gamma-ray events are observed to be associated with small energetic particle events in the interplanetary medium and vice versa.

Solar ${ }^{3} \mathrm{He}$-rich events may represent a different type of particle injection and/or acceleration process from those discussed above. Such events have ${ }^{3} \mathrm{He} /{ }^{4} \mathrm{He}$ ratios of order unity while the typical ratios for the solar atmosphere or solar wind are a few times $10^{-4}$ or less. Because the particle fluxes in ${ }^{3} \mathrm{He}$-rich events are generally quite low, these events are usually detected only by averaging over from several hours to a day. Thus the flares or other solar phenomena associated with ${ }^{3} \mathrm{He}$-rich events have been difficult to identify. Recently, however, Reames et al. (1985) have found that virtually all ${ }^{3} \mathrm{He}$-rich events are associated with impulsive $\sim 2$ to $10^{2} \mathrm{keV}$ electron events.

Most of the papers presented at this conference dealt with the analyses of new observations of energetic particles and energetic secondary emissions obtained over this solar maximum $(\sim 1980)$ by the SMM, Hinotori, ISEE, IMP, Helios and Voyager spacecraft. In this rapporteur paper I have divided the subject in to the following categories: 1) solar energetic particle events observed in space; 2) ${ }^{3} \mathrm{He}$-rich 
events; 3) solar gamma-rays and neutrons; 4) theoretical work; 5) solar neutrinos; and 6) summary. In addition the reader is referred to the invited talk by Dr. E. Chupp on gamma-ray and neutron observations from the SMM spacecraft, and a highlight talk by Dr. Stone summarizing the new developments in solar energetic par-
ticle composition.

\section{Large Solar Energetic Particle Events}

Information on the acceleration process for LSEP events is provided by measurements of the energy spectrum, elemental and isotopic composition, and charge states of the accelerated particles, and by observations of the solar phenomena associated with the acceleration.

Kahler et al. (SH 1.3-7) showed an unambiguous case of a LSEP with $>50$ $\mathrm{MeV}$ protons and relativistic electrons which was not accompanied by a solar flare or active region or any impulsive phase phenomena (Figure 1). Only a disappearing filament and classic $H \alpha$ double ribbon emission was observed on the solar disk, while a coronal mass ejection and weak interplanetary type II radio emission was observed. These phenomena suggest that the LSEP acceleration process occurred high in the corona, presumably associated with the passage of the shock wave which produced the type II radio emission. No strong complex surface magnetic fields or impulsive phase acceleration of electrons to $\sim 10-10^{2} \mathrm{keV}$ was necessary.

Measurements of the charge states, $q$, for $\sim 1 \mathrm{MeV}$ nucleon ions (Luhn et al., SH 2.1-11) in 12 LSEP events indicate that the ions come from regions with equilibrium temperatures of typically $\sim 2 \times 10^{6 \circ} \mathrm{K}$, although for a couple of elements, $\mathrm{Ne}$ and $\mathrm{Mg}$, the inferred equilibrium temperatures are higher (Figure 2). Thus the charge state measurements indicate LSEP acceleration generally occurs in the quiet corona rather than the $\sim 10^{7 \circ} \mathrm{K}$ flare plasma, consistent with the picture of acceleration by shock waves passing through the high corona. Also consistent with this picture is the complete absence of deuterium and tritium in LSEP events; ${ }^{2} \mathrm{H}$ and ${ }^{3} \mathrm{H}$ would be

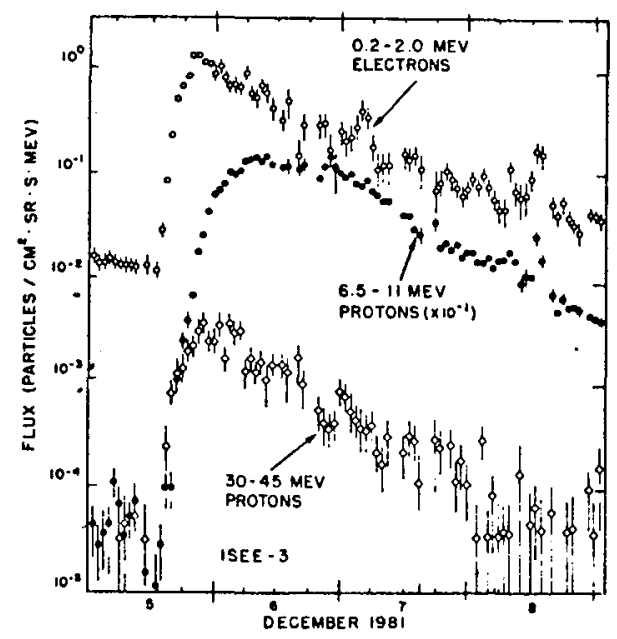

Figure 1. Flux-time plots of energetic particles for the SEP of 1981 December 5 (Kahler et al., SH 1.3-7). 


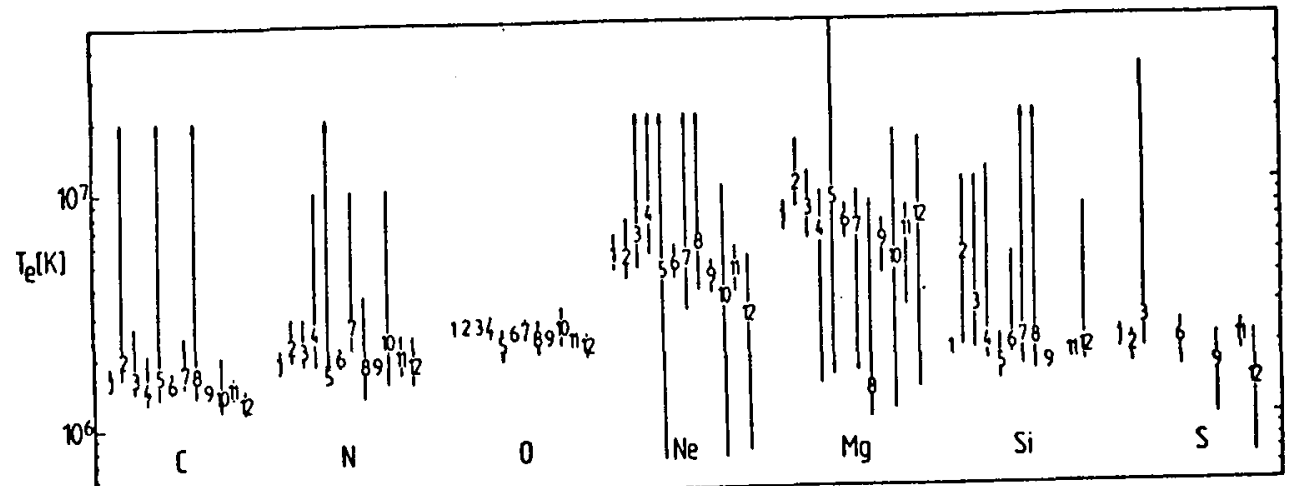

Figure 2. Equilibrium temperatures ( $95 \%$ confidence intervals or lower limits) for 12 LSEP periods (Luhn et al., SH 2.1-11).

produced by spallation if the accelerated particles had passed through significant

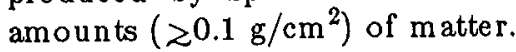

The papers by Breneman and Stone, ( $\mathrm{SH} 2.1-4,2.1-5$ ) show that the observed elemental abundances and their variation from one LSEP event to another can be understood if the charge state of each element is taken in to account. Using Luhn et al.'s (SH 2.1-11) average charge state measurements for each element, they show that the elemental abundances for a given LSEP event differ from the elemental abundances averaged over all LSEP events in a way which depends systematically on $q / m$ (Figure 3). The interpretation of this result is that the combined effects of particle acceleration and propagation gives rise to a rigidity dependence which varies from one LSEP event to another, while the underlying source elemental abundances remain essentially invariant. It is well known from previous studies (see Meyer, $1984 \mathrm{a}, \mathrm{b}$ ) that when LSEP elemental abundances are plotted versus the first ionization potential (FIP) there is a depletion compared with solar photospheric abundances for elements with a FIP above $\sim 10 \mathrm{eV}$. Breneman and Stone normalize the average elemental abundances for low FIP elements to photospheric abundances to remove the average $q / m$ dependence. The resulting normalized LSEP abundances for elements with both high and low FIP are consistent with known coronal abundances, again
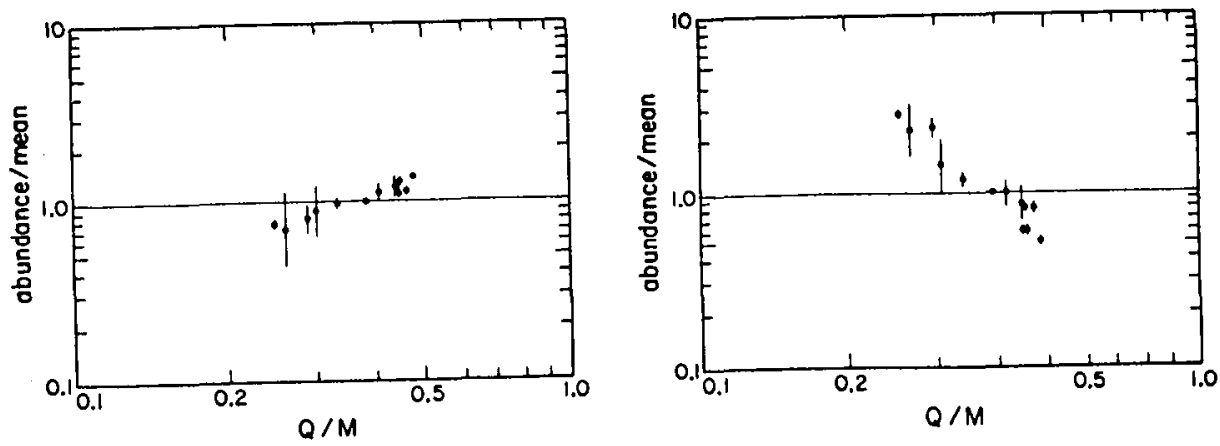

Figure 3. Abundances relative to the mean SEP abundance for two typical flares, plotted vs. $q / m$ (Breneman and Stone, SH 2.1-4). 


\section{0}

consistent with the idea of LSEP being accelerated out of normal coronal material.

The coronal structure of flares has been studied by soft X-ray (SXR) imaging telescopes aboard the Skylab (Pallavicini et al., 1977). They find that two classes of flares can be distinguished in the soft $X$-ray images: (1) impulsive, compact, lowlying $\left(\leqslant 10^{4} \mathrm{~km}\right)$ flares with small volume $\left(\sim 10^{26}-10^{27} \mathrm{~cm}^{3}\right)$, high energy density $\left(\sim 10^{2}-10^{3} \mathrm{ergs} / \mathrm{cm}^{3}\right)$, and durations of stens of minutes in soft X-rays; and (2) long duration ( hours) flares with larger volumes $\left(10^{28}-10^{29} \mathrm{~cm}^{3}\right)$, lower energy density $\left(\sim 10-10^{2} \mathrm{ergs} / \mathrm{cm}^{3}\right)$ located high in the corona $\left(\sim 5 \times 10^{4} \mathrm{~km}\right)$. These long duration SXR flares are known to accompany coronal mass ejections. Cane et al. (SH 1.2-12) find that all the LSEPs with $9-23 \mathrm{MeV}$ proton fluxes greater than $\sim 1\left(\mathrm{~cm}^{2} \mathrm{sec}\right.$ ster $\mathrm{MeV})^{-1}$ come from long duration SXR's. On the other hand the distributions of relativistic electron fluxes for the impulsive and long duration SXR types were almost the same. The LSEP's with impulsive SXR have previously been termed "electron rich" but a better label would be proton poor. A number of the LSEP's with impulsive SXR are associated with gamma-ray flares. It was noted that LSEP events with either impulsive and long duration SXR were generally $(\sim 80 \%)$ accompanied by type II radio emission indicating the presence of a shock wave. Many of the long duration SXR events were not accompanied by normal impulsive phenomena, i.e. type III or $V$ radio bursts, while the impulsive SXR events usually had accompanying type III/V
emission.

Evenson et al. (SH 1.2-14) found that the shape of the energy spectrum of relativistic electrons $\left(\sim 0.1-10^{2} \mathrm{MeV}\right)$ in LSEP events also depended on whether the accompanying SXR event was impulsive or long duration. Following the method of Lin et al. (1982), the electron spectrum was constructed by taking the electron flux at the time of maximum (TOM) for each energy interval. For diffusive propagation with negligible energy loss the TOM spectrum reflects the injection spectrum at the Sun if the spatial dependence of the diffusion coefficient is approximately the same at all energies. Long duration SXR events produced power laws in momentum in the relativistic electron spectrum, while impulsive SXR produced power laws in energy (Figure 4). Thus there appear to be clear differences in the energetic particle emission depending on the spatial structure of the associated flare phenomena at the Sun.

The energy spectrum of LSEP protons has previously been studied by McGuire and von Rosenvinge (1984) who find that the characteristic Bessel function shape which is expected from stochastic acceleration generally fits the TOM spectra from $\sim 1 \mathrm{MeV}$ to $\geq 80 \mathrm{MeV}$ (Figure 5). They note, however, strong shocks can give a similar shape at those energies. The power law in momentum observed for electrons is also consistent with shock acceleration.

\section{III. ${ }^{3} \mathrm{He}$-rich Events}

Solar ${ }^{3} \mathrm{He}$-rich events represent one of the most striking composition anomalies among the observed populations of solar and interplanetary energetic particles, with ratios of the neighboring isotopes ${ }^{3} \mathrm{He} /{ }^{4} \mathrm{He}$ of order unity. Since the last cosmic ray conference, Reames et al. (1985) have found that virtually all solar $\gtrsim 1.3$ $\mathrm{MeV}$ per nucleon ${ }^{3} \mathrm{He}$-rich events observed by the ISEE 3 spacecraft are associated with impulsive $\sim 2$ to $10^{2} \mathrm{keV}$ electron events, although many electron events were not accompanied by detectable ${ }^{3} \mathrm{He}$ increases (Figure 6). Both the ${ }^{3} \mathrm{He}$ and the electrons exhibit nearly scatter-free propagation in the interplanetary medium, and the times of onset and maximum for the ${ }^{3} \mathrm{He}$ and electron increases are closely related by 


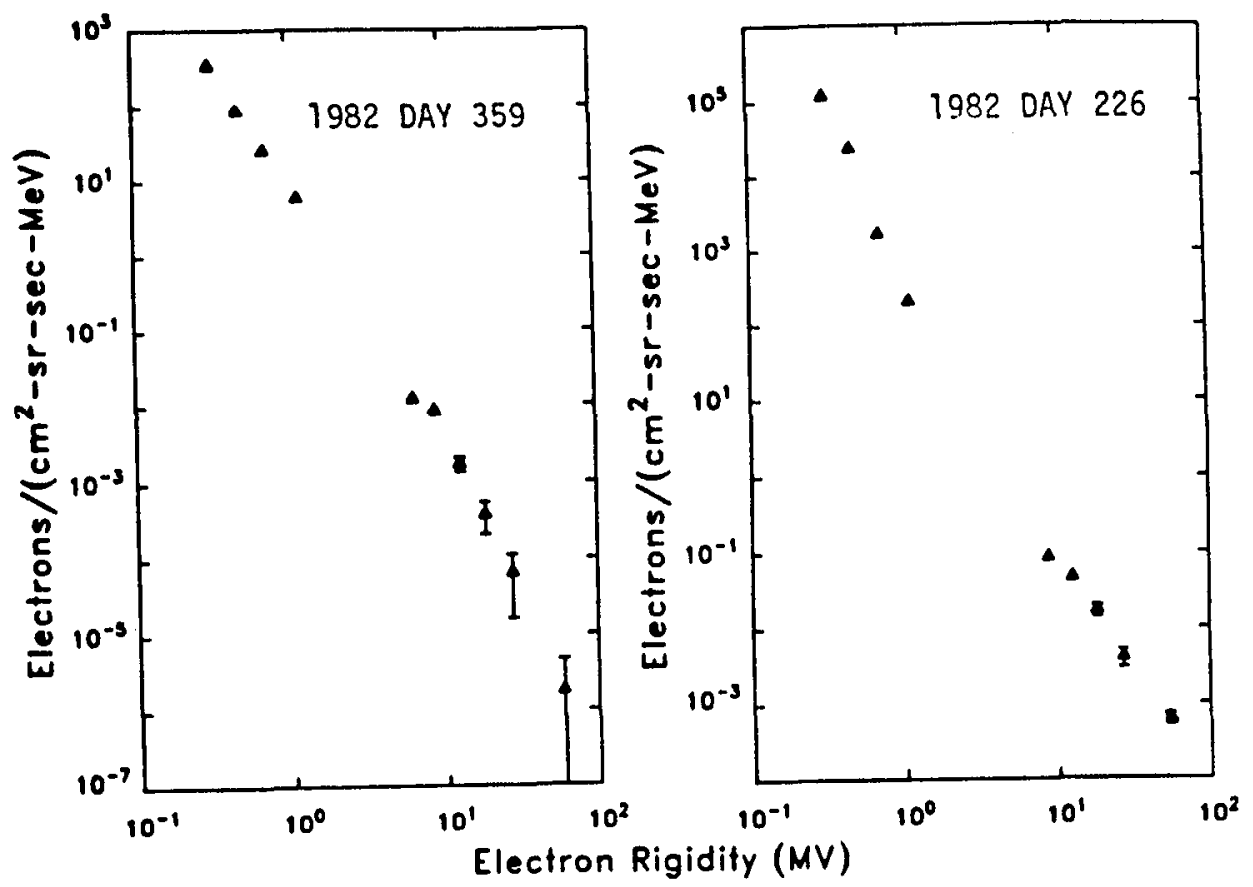

Figure 4. (a) Flare rigidity spectrum of a typical long duration SXR event. (b) Flare rigidity spectrum of a typical impulsive SXR event (Evenson et al., SH 1.2-14).

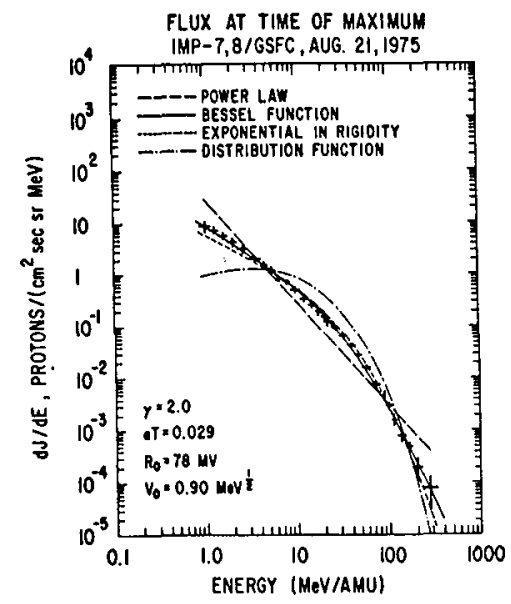

Figure 5. TOM spectrum and spectral fits for the event of 21 August 1975. The source flare was located at N26W74 (McGuire and Von Rosenvinge, 1984)

velocity dispersion. The electron events and their related type III solar radio bursts provide, for the first time, identification of the flares which produce ${ }^{3} \mathrm{He}$-rich events. Thus ${ }^{3} \mathrm{He}$ appears to be accelerated at the impulsive phase of solar flares along with nonrelativistic electrons.

Reames and Lin (SH 2.2-5) systematically studied 187 solar electron events and found ${ }^{3} \mathrm{He}$ present in over half of the events. They suggest that ${ }^{3} \mathrm{He}$ would be 


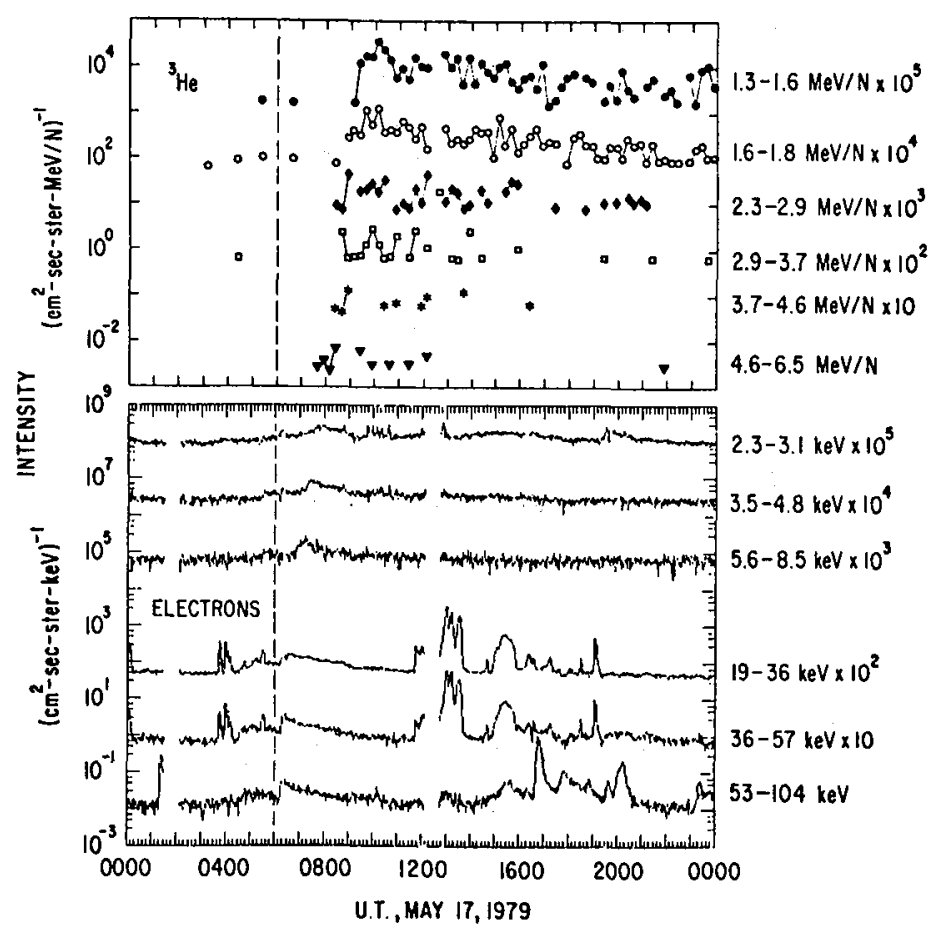

Figure 6. Time histories of the intensities of ${ }^{3} \mathrm{He}$ and electrons of the indicated energy during $1979 \mathrm{May}$ 17. The appearances of the plots differ partly because of the absence of continuous background fluxes for ${ }^{3} \mathrm{He}$. The lowest isolated points for each energy of ${ }^{3} \mathrm{He}$ represent one particle per 15 minute averaging period. The rapidly varying bursts (at $\sim 1200 \mathrm{UT}$ ) observed in the higher, $19 \mathrm{keV}$, energy electron channels are due to particles from Earth's bow shock. The 53$104 \mathrm{keV}$ channel also responds to solar X-rays ( 0130,1630-2030, and 2330 UT). The dashed vertical line indicates the time of the Type III solar burst. Intensity scale factors are shown to the right of the energies (Reames et al., 1985).

found in all electron events if the ${ }^{3} \mathrm{He}$ detection sensitivity were better. Reames and Stone (SH 2.2-3) showed that in the absence of electron data, the kilometric wavelength type III bursts could be used to identify the associated flare event at the Sun. One of the events so identified is also a gamma-ray flare event. Kahler et al. (SH 2.2-4) studied the solar source of the ${ }^{3} \mathrm{He}$-rich events identified by Reames et al. (1985) and suggest that the particle acceleration may be occurring high in the corona above the $\mathrm{H} \alpha$ flare.

Luhn et al. (SH 2.2-8) measured the mean ionic charge of silicon measured over $22{ }^{3} \mathrm{He}$-rich periods in $1978-79$ to be $\bar{q} \approx 14$, i.e. essentially fully ionized. The ionic charge state of iron for ${ }^{3} \mathrm{He}$-rich flares had been previously reported to be $\bar{q} \approx 20.5$. These values are inconsistent with resonant heating by harmonics of Hecyclotron waves (Fisk, 1978), but are consistent with the source region for ${ }^{3} \mathrm{He}$-rich flares having a temperature of $\sim 10^{7 \circ} \mathrm{K}$, well above the $\sim 2 \times 10^{6 \circ} \mathrm{K}$ temperature inferred for normal LSEP flares.

Mason et al. (SH 2.2-7) surveyed the elemental abundances for $66{ }^{3} \mathrm{He}$-rich flares observed by ISEE 3 in 1978-82 (Figure 7). It is known that He-rich flares show a tendency to be enriched in heavy ions. The enhancements over normal LSEP 


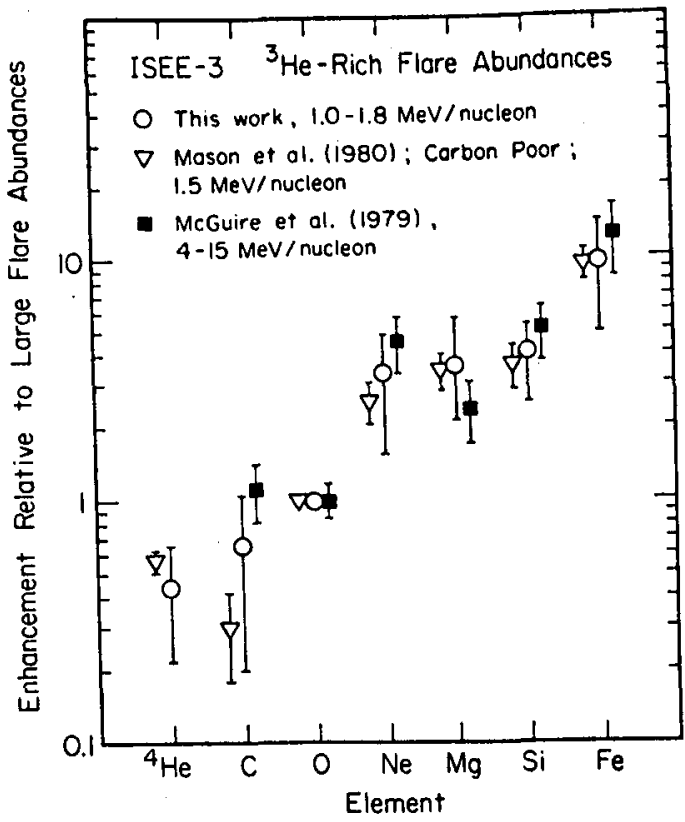

Figure 7. From Mason et al. (SH 2.2-7).

abundances increase with $A$ or $Z$ from He to Fe. Mason et al. show that the heavy ion abundances do not vary very much from flare to flare compared with the variation in the ${ }^{3} \mathrm{He} /{ }^{4} \mathrm{He}$ abundance. Furthermore the heavy ion abundances do not vary much with energy over the measured range of $\sim 1$ to $15 \mathrm{MeV} / \mathrm{nucleon}$. This lack of significant variation in heavy ion abundances suggests that heavy ion enrichments are present in the ambient source plasma where ${ }^{3} \mathrm{He}$-rich flares occur.

When ${ }^{3} \mathrm{He}$-rich events are compared with normal LSEP events in, a consistent picture seems to emerge. ${ }^{3} \mathrm{He}$-rich events occur in hot plasmas, $z 10^{7 \circ} \mathrm{K}$, where most heavy elements will be fully ionized. Because $q / m$ would then be $\sim 1 / 2$ for essentially all the measured heavy ions, the acceleration mechanism, which appears from LSEP events to be rigidity dependent and highly variable from flare to flare, does not alter the source composition. The high temperature, $\sim 10^{7 \circ} \mathrm{K}$, and consistent pattern of heavy ion enrichment (which must include a significant ${ }^{4} \mathrm{He} / \mathrm{H}$ enrichment so the ${ }^{3} \mathrm{He}$ can be preferentially heated via Fisk's cyclotron resonance mechanism) are clues to the region of the solar atmosphere where the acceleration takes place. The fact that these ${ }^{3} \mathrm{He}$-rich events appear to be closely associated with impulsive phase electron events suggests that the ${ }^{3} \mathrm{He}$ acceleration process is the same as that which accelerates the $\sim 1-10^{2} \mathrm{keV}$ electrons in the impulsive phase of solar flares. It may be significant that very few ${ }^{3} \mathrm{He}$-rich events are accompanied by shock waves.

\section{Gamma-rays and neutrons}

Accelerated protons, alphas, and heavier nuclei with energies above $\sim 10$ $\mathrm{MeV}$ produce gamma-ray lines and neutrons via interactions with the solar atmosphere. Energetic electrons produce hard $\mathrm{X}$-ray and gamma-ray continuum via 


\section{4}

bremsstrahlung. Measurements of gamma-rays and neutrons provide unique information on the nuclear acceleration and interaction processes at the Sun. Since 1980 the SMM and Hinotori spacecraft have provided a wealth of new data on solar gammarays, neutrons, and other high energy neutral emissions. The results have been summarized by Dr. Chupp in his invited talk. The main points pertaining to the acceleration of particles at the Sun are:

1) Both electrons and ions are often accelerated together in the impulsive phase of the flare.

2) The acceleration can occur in seconds for both electrons and ions.

3) The maximum energies reached by the acceleration is in the $\mathrm{GeV}$ range for ions and $\sim 10^{2} \mathrm{MeV}$ for electrons.

4) The relativistic electrons appear to exhibit evidence for directivity.

5) The spectrum of the accelerated ions at the Sun appears to show a steepening with energy which is consistent with a Bessel function shape comparable to those observed for LSEP events (Figure 5). The proton fluxes at the Sun inferred from the gamma -ray and neutron observations, however, are poorly correlated to the escaping LSEP fluxes observed in interplanetary space.

6) There is approximate proportionality between the 4-7 MeV continuum flux, which is primarily nuclear in origin, and the $>0.27 \mathrm{MeV}$ electron bremsstrahlung continuum flux. Chupp interprets this correlation to mean that ions may be accelerated to $z 10 \mathrm{MeV}$ in every flare. Only relatively intense flare events, however, are detectable by the nuclear gamma-ray measurements and to a lesser extent, by the $>0.27 \mathrm{MeV}$ bremsstrahlung continuum measurements. Bai and Dennis (1985), using had X-ray $(>30 \mathrm{keV})$ data, argue that the gamma -ray line flares have characteristics which distinguish them from normal flares.

Neutrons with energies $\sim 1 \mathrm{GeV}$ have been detected by the Jungfraujoch ground-based neutron monitors from the 3 June 1982 flare, while protons from the decay of solar neutrons have been detected for three separate flares by the ISEE 3 spacecraft (Evenson et al., SH 1.2-4). This latter type of measurement gives the most accurate neutron energy spectrum since essentially all of the neutron energy is carried by the decay proton.

McDonald et al. (SH 1.3-8), Van Hollebeke et al. (SH 2.1-3), and Neustock et al. (SH 1.3-9) used data from the Helios I spacecraft to examine the energetic solar particles associated with the gamma-ray flares. The Helios I spacecraft was located close to the Sun, $50.5 \mathrm{AU}$, and close in heliolongitude to the flare site for the gamma-ray flares of 7 and 21 June 1980 and 3 June 1982, so any escaping particles should be easily detectable. McDonald et al. and previously McDonald and Van Hollebeke (1985) note that small precursor energetic particle events were observed several hours prior to all three of these gamma-ray flares (these precursor events would not be detectable at 1 $\mathrm{AU}$ ), and thus the acceleration of an existing reservoir of stored energetic (up to 60 $\mathrm{MeV}$ ) particles could be involved in these gamma-ray flares. In support of models of re -acceleration it should be noted that the spectrum of the ions in those LSEP events is unusually hard (Figure 8). For the 3 June 1982 event, the spectrum of the escaping protons (power law exponent $\sim 1.2$ up to $200 \mathrm{MeV}$ ) appears to be inconsistent 

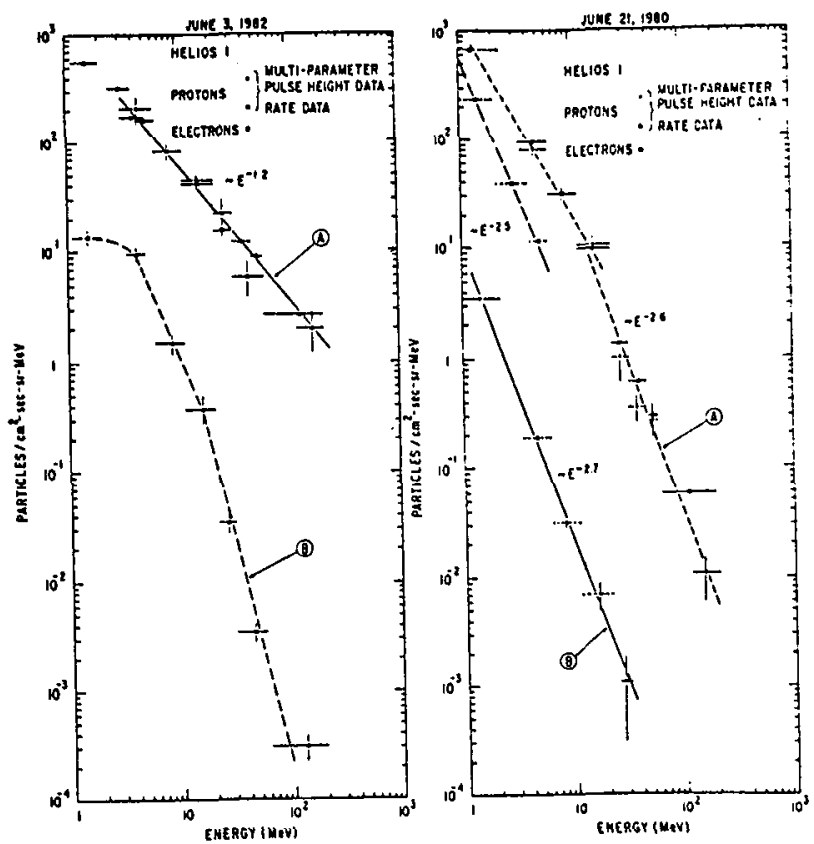

Figure 8. Energy spectra measured at the time of peak intensity for each energy interval. The measured particle anisotropy at this time is still $\sim 60 \%$, so convective equilibrium has not been established. For each panel the upper plot is the spectral data for the main event, and the lower data set is for the precursor event (McDonald and Van Hollebeke, 1985).

with the spectrum inferred from the gamma-ray and neutron observations of the impulsive phase and may require extended acceleration (Ramaty and Murphy, 1985). Chupp et al. (SH 1.4-1) and Forrest et al. (SH 1.4-7) show evidence from SMM for the extended production of both neutrons and pions for $z^{5}$ minutes beyond the impulsive phase for the flare of 3 June 1982 while electron bremsstrahlung at energies above $\sim 10$ $\mathrm{MeV}$ was observed only during the impulsive phase (Figure 9). The additional acceleration after the impulsive phase may have produced the hard proton spectrum observed at Helios I; Forrest et al. point out that $\sim 80 \%$ of the neutral pion decay photons were observed after the impulsive phase.

McDonald et al. also point out that significant amounts of ${ }^{3} \mathrm{He}$ are observed for 3 June 1082 and 21 June 1980 as well as unusually high abundances of Fe relative to oxygen. This $\mathrm{Fe} / \mathrm{O}$ enhancement is comparable to that observed for ${ }^{3} \mathrm{He}$-rich events.

Neustock et al. (SH 1.3-9) studied the time of injection of electrons and ions on 7 June 1980. The particle fluxes were highly anisotropic, streaming along the magnetic field away from the Sun. The travel time for each particle species was subtracted to obtain a solar release time (SRT). For each of the three major flares $(0117,0302$, $0725 \mathrm{UT}$ ) the $\sim 0.5 \mathrm{MeV}$ electron injection began promptly (within one minute) at the impulsive flare hard $\mathrm{X}$-ray burst time but lasted at least several minutes longer. The 3-20 $\mathrm{MeV}$ protons were injected promptly at the time of the third flare, but showed three separate delayed "injections" $(0345,0440,0635$ SRT) for the second 

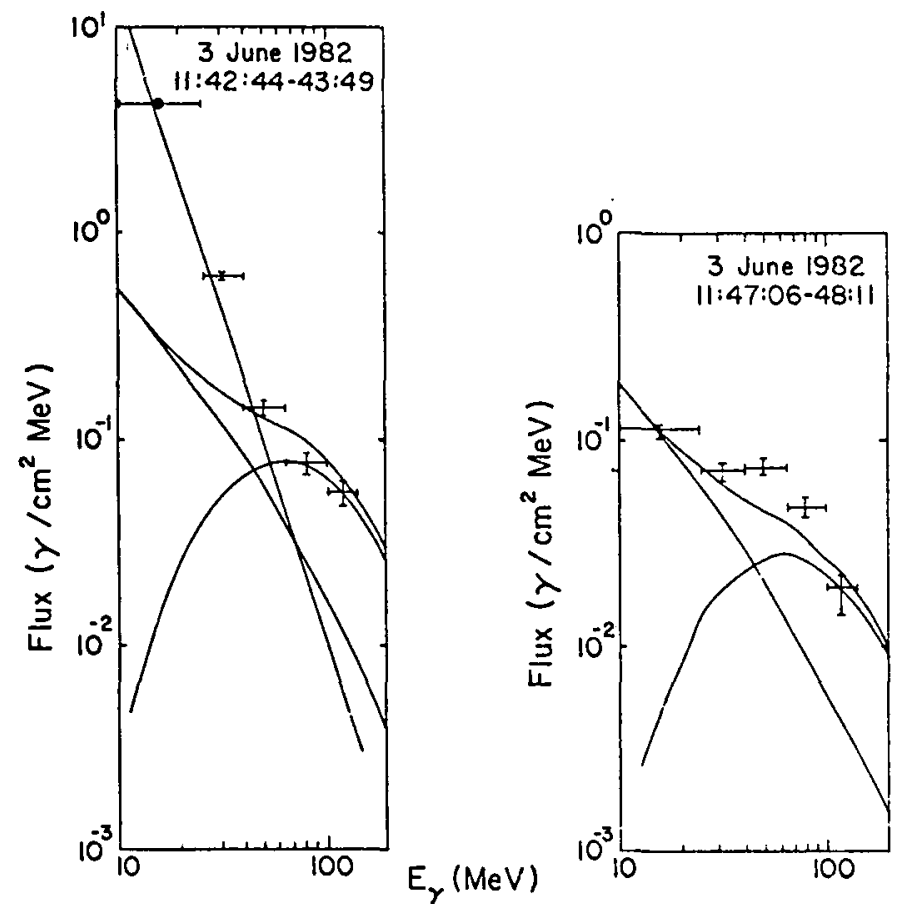

Figure 9. (a) The 10-140 MeV photon spectrum for the impulsive phase of the 3 June 1982 flare. The steeply falling line is a power-law fit to the electron bremsstrahlung component; the other curves are fits to the neutral pion decay component. (b) The 10-140 MeV photon spectrum for the extended phase, $\sim 5$ minutes after the impulsive phase. The spectrum is consistent with a pure pion decay origin without any electron bremsstrahlung component (Forrest et al., SH 1.4-7).

flare (Figure 10). These delayed "injections" showed no velocity dispersion, i.e. protons of all energies were seen at Helios at the same time. One possible interpretation is that the second flare probably filled only certain channels of interplanetary field lines with energetic protons, which were then crossed by Helios. Similar channels

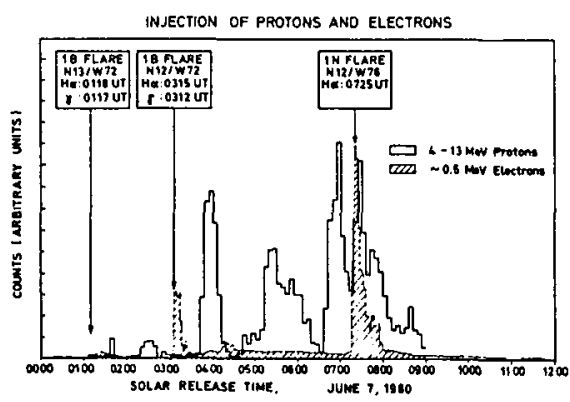

Figure 10. Solar injection of electrons and protons for the 7 June 1980 event, after correction for interplanetary travel time along the smooth interplanetary magnetic field. Solar release Time $(\mathrm{SRT})=-s / v$ (Neustock et al., SH 1.3-9). 
have been identified in ISEE 3 solar electron measurements at 1 AU (Anderson and Dougherty, SH 3.2-3). Finally, only the first two flares had detectable $>0.3 \mathrm{MeV}$ gamma-ray emission, and only the second emitted nuclear lines, but the third flare gave rise to the most in tense particle event at Helios I. These fairly direct measurements of the energetic particles escaping from gamma-ray flares suggest that if the escaping and gamma-ray producing particle populations are produced by the same acceleration, then the height of the acceleration region must vary from flare to flare.

Gamma-ray line measurements provide a new method to obtain solar elemental abundances. Narrow lines are produced by the interactions of energetic protons and alphas with ambient heavy nuclei. Since the nuclear processes which produce gamma-ray lines are essentially unaffected by ambient conditions, and the theory and cross-sections for these processes are well-known, measurements with high spectral resolution $\left(\Delta E / E \sim 10^{-3}\right)$ could provide accurate abundance determinations for the solar atmosphere. Even with the relatively poor spectral resolution $(\triangle E / E \sim 7 \%)$ of the SMM GRS instrument, Murphy et al. (SH 2.1-13 and -14) were able to determine the abundances of $\mathrm{C}, \mathrm{O}, \mathrm{Ne}, \mathrm{Mg}, \mathrm{Si}, \mathrm{Fe}$ and $\mathrm{O}$ to $\pm 10-30 \%$, and to show that they are significantly different from local galactic (solar) abundances, but consistent with coronal abundances.

\section{Theoretical work}

Since the gamma-ray measurements by SMM showed that ions are of ten accelerated to energies of well above $\sim 100 \mathrm{MeV}$ nearly simultaneous (with in seconds) with the impulsive phase electrons, one of the key questions has been whether stochastic shock acceleration models are rapid enough. The theoretical contributions reported here suggest that shock acceleration can accelerate ions to $>10 \mathrm{MeV}$ energies in times $\leqslant 1$ s. Decker and Vlahos (SH 1.1-6) show by numerical simulations of their turbulent oblique shock model that protons can be accelerated from a $100 \mathrm{keV}$ injection energy to $\gtrsim 10 \mathrm{MeV}$ in as short a time as $\sim 6 \mathrm{msec}$ when the shock is quasiperpendicular. Ellison and Ramaty (SH 1.1-5) find that their first order Fermi shock acceleration model can reproduce the observed LSEP energy spectra for electrons, alphas, and protons for a given flare with a single shock compression ratio. They also find that the acceleration time up to $\sim 100 \mathrm{MeV}$ can be as short as $\sim 1 \mathrm{~s}$ from an assumed injection energy of $\sim 100 \mathrm{keV}$. Droge and Schlickeiser (SH 1.1-4) propose a combination of first and second order Fermi acceleration in shock waves. They are able to reproduce the observed correlation ( $L$ in et al., 1984) between the high and low energy spectral indices in the double power law spectrum of electrons, and the correlation between electron low energy spectral indices and proton spectral indices. As in the other models an initial injection energy is assumed; here it is $50 \mathrm{keV}$.

The question of the injection of low energy ions may be important for fiare energetics as well. Low energy $\sim 10-100 \mathrm{keV}$ electrons can be observed at the Sun via the bremsstrahlung $\mathrm{X}$-rays they produce. Ions below $\sim 10 \mathrm{MeV}$, however, are essentially invisible at the Sun. Simnett (SH 1.2-13) argues that protons of $\sim 10-1000$ $\mathrm{keV}$ rather than electrons may in fact contain most of the flare energy. Computations by Canfield and Chang ( $\mathrm{SH} 1.3-5$ ) indicate that it may be possible to detect $\sim 10-10^{3}$ $\mathrm{keV}$ protons which are beamed downward into the solar atmosphere, via the Doppler shifted Lyman alpha radiation they produce in the process of electron pickup and loss. Clearly information on this component would be very useful. 


\section{Solar Neutrinos}

The solar neutrino experiment of Davis et al. (1983) has now been taking data for more than fifteen years with the well known result that the observed average flux of solar ${ }^{8} \mathrm{~B}$-neutrinos is only about $1 / 3$ of that expected from standard solar models. The measurements are taken over periods of several months at a time and although the uncertainties in the data points are very large, it is remarkable that the three in tervals with the largest production rates include the largest solar energetic particle events observed over that period (Figure 11). The expected production of neutrinos by the observed solar energetic particles themselves, either at the Sun or at the earth, falls many orders of magnitude below the observed increases. There remains a possibility that some process associated with very large energetic particle flares occurs beneath the photosphere to produce an increase in the neutrino flux. It would be highly desirable to determine if the association of high neutrino count rates with the largest LSEP's is in fact statistically significant.

\section{Summary}

It is highly likely that the acceleration of LSEP particles is due to the passage of shock waves through the quiet corona. For a significant number of LSEP events no impulsive phase is observed. This suggests that injection of particles at suprathermal energies $(50-100 \mathrm{keV})$ is not required. The acceleration process might thus be similar (but scaled up from) that observed at the earth's bow shock, where reflection of a small fraction of the solar wind thermal particles by the jump in the magnetic field at the shock apparently provides the seed population for further acceleration, which may occur via a Fermi process (see J. Geophys. Res., volume 86, number A6). The spatial structure of the related solar flare or transient appears to play an important role in the acceleration process, both in determining the energetic particle fluxes which escape to the interplanetary medium and, for electrons, their energy spectrum.

The acceleration process in ${ }^{3} \mathrm{He}$-rich events appear to be distinctly different. It occurs in regions of high, $210^{7 \circ} \mathrm{K}$, temperatures with a characteristic pattern of enhancement of the abundances of heavy elements from He to Fe. The acceleration

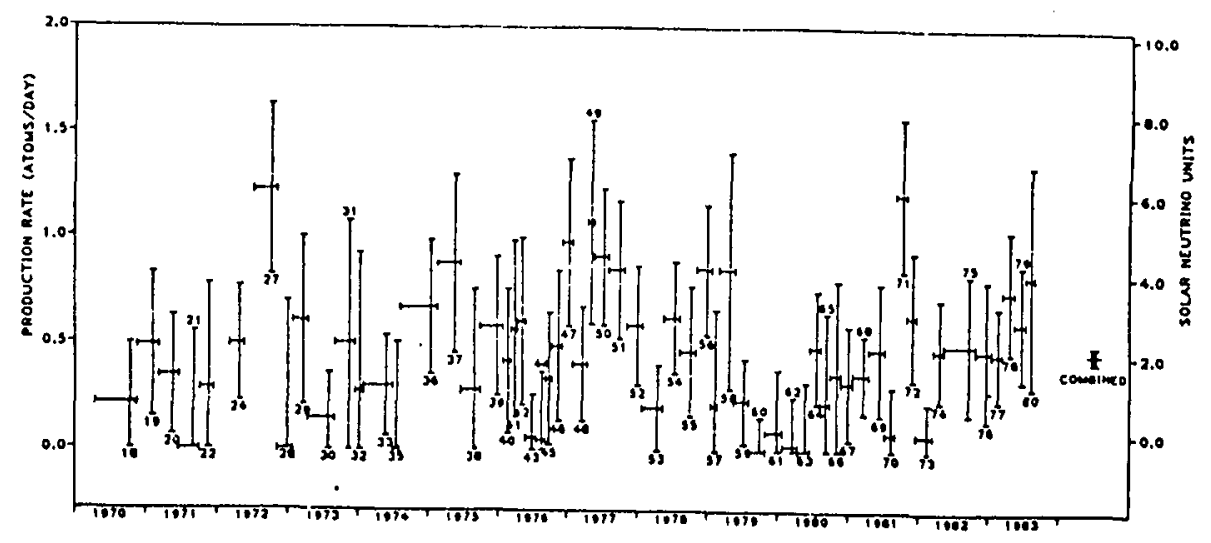




\section{8}

process also accelerates electrons to non-relativistic $10-10^{2} \mathrm{keV}$ energies, and occurs during the impulsive phase of flares. This acceleration appears not to be shock related.

Solar gamma-ray events are generally associated with flares having intense impulsive phase phenomena, and often with impulsive, compact soft $X$-ray flare sources. Shock waves (i.e., type II radio emission) generally are observed as well. Often some ${ }^{3} \mathrm{He}$ and heavy element enrichments are observed from gamma-ray flares. It seems plausible that shock waves passing through low -lying compact flare structures result in an intense, rapid second -step acceleration which leads to the highly energetic ions and electrons required for the gamma -ray production. Such second step acceleration is suggested by the short delays $(\sim 1 \mathrm{~s})$ that are commonly observed in the $\gtrsim 100$ $\mathrm{keV}$ hard $\alpha$-ray emission for gamma-ray flares (Bai and Dennis, 1985).

Gamma -ray and neutron observations from SMM and Hinotori have proved to be a powerful complement to detailed energetic particle measurements in interplanetary space for probing solar particle acceleration mechanisms. Furthermore, both types of measurements show great potential for providing highly accurate elemental and isotropic abundance measurements for the Sun. Powerful new observational techniques for measurement of both particles and photon emissions are now available to provide the next leap forward, and it is my hope that the opportunity to make that leap will come in the next solar maximum.

\section{Acknowledgments}

I wish to acknowledge useful discussion with E. Stone, R. Murphy, F. McDonald, G. Wibberenz, and R. McGuire, among others. This research was supported in part by NASA grant NAG 5-376.

\section{References}

Bai, T., and B. Dennis, 1985, Astrophys. J., 292, 699.

Davis, R., Jr., B. T. Cleveland, and J. K. Rowley, 1983, Science Underground, AIP Conf. Proc. No. 96, p. 2 (ed. M. M. Nieto et al.), Amer. Inst. Phys., New York.

Fisk, L. A., 1978, Astrophys. J., 224, 1048.

Lin, R. P., R. A. Mewaldt, and M. A. I. Van Hollebeke, 1982, Astrophys. J., 25s, 949.

McDonald, F. B., and M. A. I. Van Hollebeke, 1985, Astrophys. J., 290, L67.

McGuire, R. E., and T. T. Von Rosenvinge, 1984, Adv. Space Res., 4, 117.

Meyer, J.-P., 1985a, Astrophys. J. Suppl., 57, 151.

Meyer, J.-P., 1985b, Astrophys. J. Suppl., 57, 173.

Pallavicini, R., S. Serio, and G. S. Vaiana, 1977, Astrophys. J., 217, 108.

Ramaty, R., and R. J. Murphy, 1985, Adv. Space Res., 4, 127.

Reames, D. V., T. T. Von Rosenvinge, and R. P. Lin, 1985, Astrophys. J., 292, 716. 